\title{
MASTERCLASS
}

\section{Liver disease in twins with Felty's syndrome}

\author{
R J Moots, E Elias, S Hubscher, M Salmon, P Emery
}

In 1924 Felty reported the association of rheumatoid arthritis (RA), splenomegaly and neutropenia. ${ }^{1}$ Other features including thrombocytopenia, anaemia, generalised lymphadenopathy, skin ulcers and hyper pigmentation have been added and it has become generally accepted that Felty's syndrome (FS) is an uncommon but severe variant of RA, developing in approximately $1 \%$ of patients. ${ }^{2}$ Affected patients tend to have a severe, destructive arthritis despite little evidence of objective synovitis, and exhibit a higher frequency of extra-articular manifestations, including vasculitis. Although the aetiology of FS, in common with RA, is unknown, over $90 \%$ of patients are HLA-DR4 positive, compared with $70 \%$ of patients with RA and only $30 \%$ of controls, suggesting a genetic basis: ${ }^{3}$ indeed, a striking proportion are homozygous for the HLA-Dw4 allele. ${ }^{4}$ In this article liver disease, one of the less well-known associations of FS, is discussed, and stresses the clinical features, investigations, aetiopathology, and management.

\section{Case histories}

TWIN 1

This 45 year old woman developed RA at the age of 16 , with an acute, severe onset. The subsequent course of her articular disease was characterised by mild synovitis, but widespread deformity. By the age of 25 she had developed symptomatic secondary Sjögrens syndrome and had weakly positive anti-Ro and anti-La auto antibodies. Five years later, aged 30 she became hypothyroid with anti-thyroid microsomal auto antibodies. At 31 years old she developed FS, detected by routine hospital monitoring and within one year had a splenectomy for severe neutropenia and thrombocytopenia, after which her white cell count and platelets rose to normal. Before her splenectomy she had mildly abnormal liver function tests, with aspartate transaminase (AST) and alkaline phosphatase (AlkP) elevated to nearly three times the upper limit of normal. A liver biopsy was performed which showed scanty non-caesating granulomas (fig 1). Despite a full investigation, there was no evidence for any systemic disease associated with granulomas. Her arthritis was well-controlled on methotrexate and she was systemically well. Her liver function tests spontaneously returned to normal before splenectomy and have remained normal.
TWIN 2

The identical twin of this woman developed seropositive, erosive RA at the age of 21 with a similar acute, severe onset, progressing to nearly identical deformities to her sister. She also developed symptomatic secondary Sjögrens syndrome with weakly positive Ro and $\mathrm{La}$ antibodies at the age of 25; and although not yet hypothyroid, she had antithyroid microsomal auto antibodies by the age of 30 . At the age of 43 , her white cell count fell from normal to less than $1.5 \times 10^{9} / 1$ with a hyper-cellular marrow and she was noted to have splenomegaly, and therefore FS. Over the next year her white cell count fell to less than $1.5 \times 10^{9} / 1$. Her joints, were, however, inactive. At this time, liver function tests were mildly abnormal with alkaline phosphatase 24 (normal range 3-12 u/l) and aspartate transaminase $46 \mathrm{u} / 1(5-35 \mathrm{u} / \mathrm{l})$. Ultrasound examination of the abdomen showed a large spleen and normal liver with normal ducts. Liver biopsy was performed and histological examination revealed nodular regenerative hyperplasia (fig 2). In November 1992 she was assessed for possible splenectomy and underwent hepatic venous pressure measurements. These showed a wedged hepatic venous pressure (WHVP) of $21 \mathrm{~mm} \mathrm{Hg}$, with a free hepatic venous pressure (FHVP) of only $2 \mathrm{~mm} \mathrm{Hg}$ (fig 3). This gradient of $19 \mathrm{~mm} \mathrm{Hg}$ (normal less than 12) was of the order seen in patients with portal hypertension due to cirrhosis of the liver and variceal bleeding.

Later that month she had an uneventful splenectomy. Six months after the operation, however, she presented with haematemesis and melaena. Endoscopy at that time showed two small varices in the oesophagus and a large gastric varix with an overlying punctum, the latter being the presumed site of the bleeding. Transhepatic venography was repeated. The portal vein pressure was again elevated at $23 \mathrm{~mm} \mathrm{Hg}$. A coronary vein feeding gastric and oesophageal veins was observed, shunting blood via the gastric veins to the left renal veins and inferior vena cava. Because of this, the coronary vein was embolised with three $15 \mathrm{~mm}$ coils and one 8 $\mathrm{mm}$ coil. Since this procedure, she has remained well and has had no further bleeding episodes. A recent endoscopy has demonstrated only one very small oesophageal varix of considerably smaller proportions to those noted previously. 


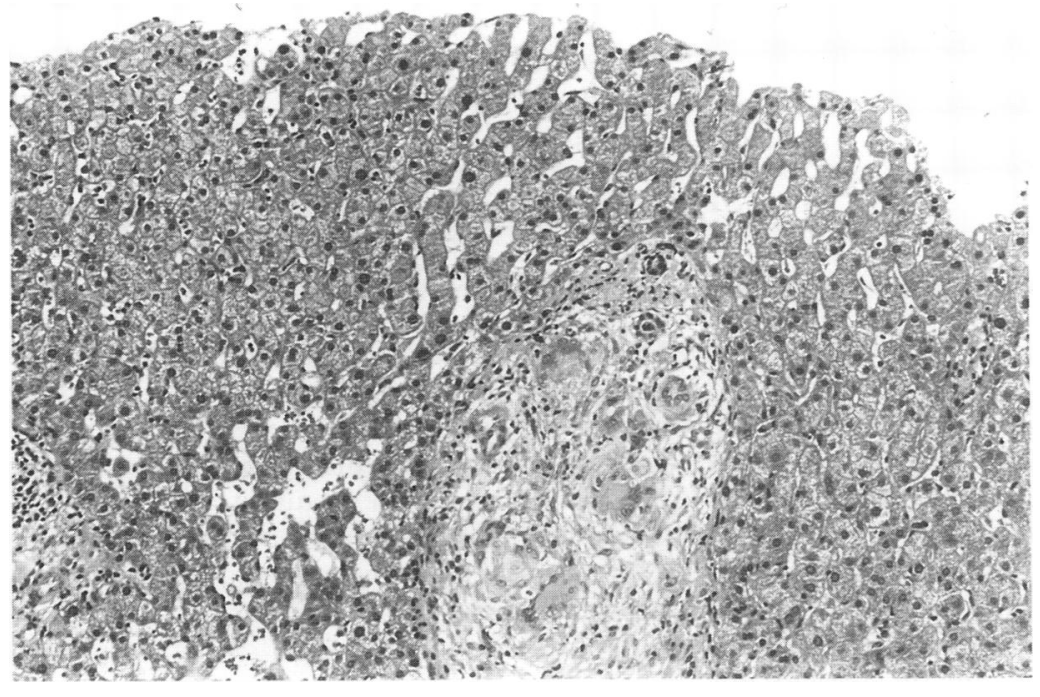

Figure 1 Haematoxylin and eosin stain of liver biopsy from twin 1. There are noncaeseating granulomas around a portal tract. The adjacent liver parenchyma is unremarkable.
A
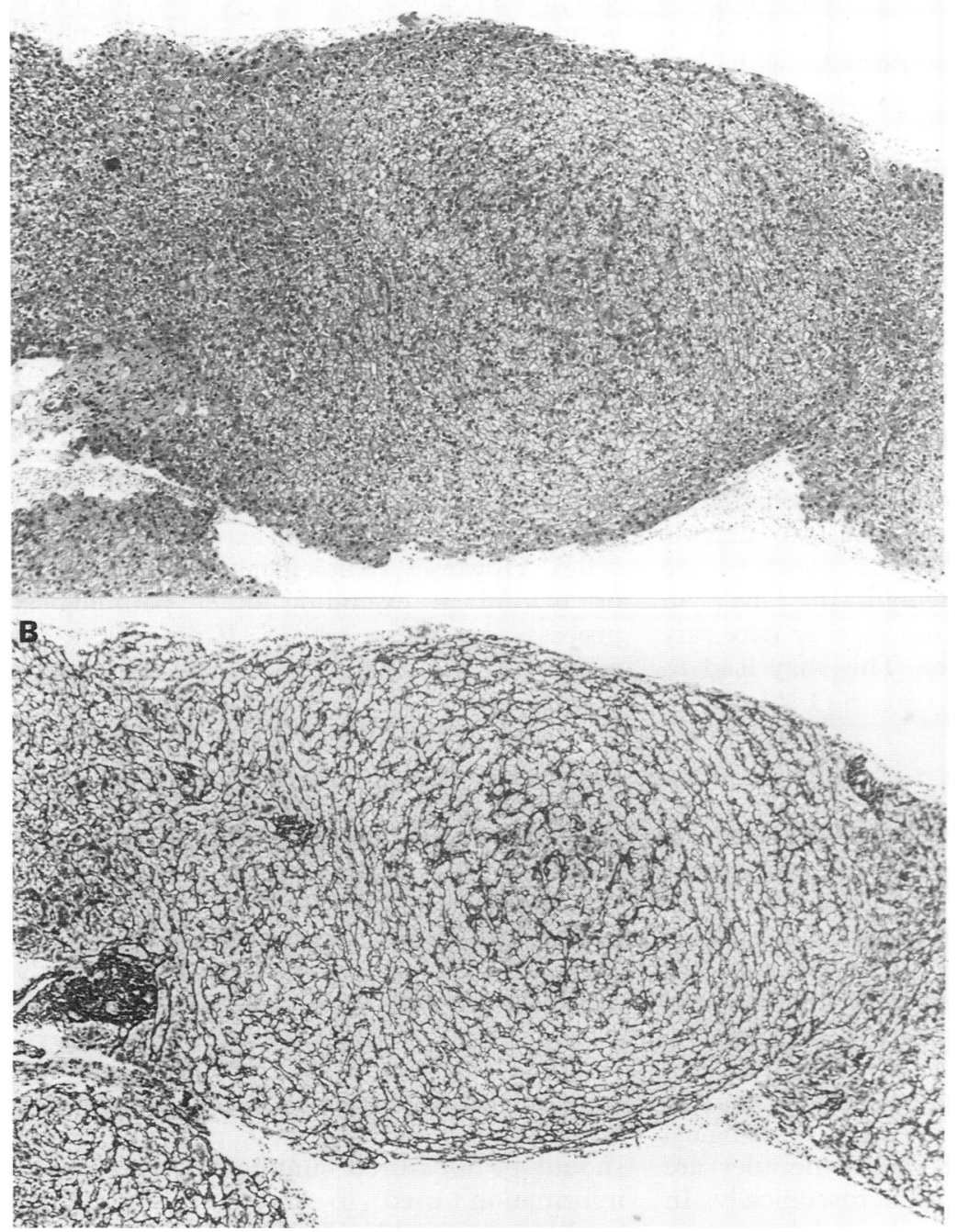

Figure $2 A$ Haematoxylin and eosin stain of the liver biopsy from twin 2. There is possibly a vague impression of nodularity; $2 B$ Reticulin staining of the the same biopsy (twin 2).

This accentuates the nodularity, with compression of cell plates at the periphery of $a$ hyperplastic nodule.
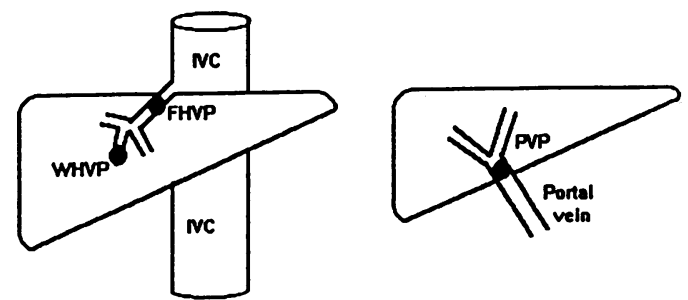

Figure 3 The wedged hepatic venous pressure gradient (WHVG = WHVP-FHVP) is the most commonly used measure of portal hypertension in clinical practice. In alcoholic cirrhosis, WHVP and portal venous pressure (PVP) are equivalent, but in patients such as ours with NRH, WHVP (21 mm Hg pre-splenectomy) may have underestimated directly measured PVP $(23 \mathrm{~mm} \mathrm{Hg}$ post splenectomy). In this diagram, the black dots indicate the positions where pressure recordings may be obtained.

\section{Discussion}

GENETICS AND FELTY'S SYNDROME

Although there is a well known genetic association between susceptibility to RA and the HLA-DR4 gene in the MHC class II region, there are clearly environmental factors contributing to this disorder: the HLA-linked contribution has been calculated to exert probably no more than $30 \%$ of the total genetic effect. ${ }^{5}$ In FS, the frequency of HLA-DR4 is of the order of $95 \%$, and there is preferential association with the Dw4 subtype of HLADR4. ${ }^{4}$ Such a strong genetic association is wellillustrated by the almost perfect concordance of the clinical courses of the two sisters here: however, although they are concordant for almost all of their disease and both had liver disease, they differed with respect to the type of liver disease. $\mathrm{NRH}$, a granulomatous disease, which had been proposed to be genetically determined, is therefore dependent on additional environmental factors.

\section{NEUTROPAENIA AND FS}

There are a number of possible causes for low white cell counts in patients with RA. Bone marrow suppression affecting white cells may occasionally occur as a result of RA per se, but is more usually found as a consequence of second-line drugs. The mechanism for neutropenia may be by direct marrow suppression, or rarely by peripheral breakdown mediated by auto antibodies. Hypersplenism is certainly an important contributor, but the depression of white cell count is often too great to be attributed to hypersplenism alone. Antineutrophil antibodies have been found in FS, and direct marrow-suppressive effects of the large infiltrate of $\mathrm{T}$ cells often found in FS may also contribute to the neutropaenia. ${ }^{6}$

Serious neutropenia in RA always merits full investigation. Bone marrow biopsy is useful to distinguish between underproduction (hypo cellular marrow) and peripheral consumption (hypercellular marrow) of white cells, as in the case of twin 2 .

\section{DIFFERENTIAL DIAGNOSIS OF LIVER}

ABNORMALITIES IN FS

Liver disease in FS, as in any other condition, 
Diseases with increased incidence of NRH

Rheumatoid arthritis

Felty's syndrome

Scleroderma

Scleroderma

Polymyalgia rheumatica

Polymyalgia rheumatica

Systemic lupus erythematosus

Waldenstrom's macroglobulinaemia

Wodgkin's disease

Hodgkin's disease
'Normal pupulation', Age $>80$

requires detailed investigation including biopsy where appropriate. The cause of the granulomas in twin 1 was never found, but as she spontaneously recovered within a matter of weeks, the problem was not severe. Of course any sort of liver disease may occur in FS as a coincidental finding and such patients may be at particular risk of reactivation of Mycobacteria tuberculosis because of immunosupression from therapy, or FS per se (in practice this is very uncommon). Methotrexate mediated liver damage is also rare when appropriate drug monitoring measures are undertaken.

There is, however, a liver abnormality with a particularly strong association with FS, namely nodular regenerative hyperplasia (NRH) of the liver (fig 2). In one study, 35\% of patients with FS and liver function abnormality had histological evidence of NRH. $\mathrm{NRH}$ has also been reported, with less frequency, in association with other conditions (table) and may be found in the livers of patients aged more than 80 years with no associated disease ${ }^{7}$ This condition was found to be the cause of the liver function abnormalities in twin 2 .

HISTOLOGY OF NRH

In NRH, the liver retains a basically normal architecture, but contains nodules of hyperplastic hepatocytes alternating with zones in which liver cell plates are compressed (fig 2). There is no fibrosis, in contrast to hepatic cirrhosis. Such histological appearances are typically present throughout the liver in patients with NRH, but the severity may vary from one area to another. This may lead to sampling difficulties.

\section{AETIOLOGY OF NRH}

Although the aetiology of NRH has not yet been fully defined, detailed histological studies have demonstrated obliterative lesions in portal vein branches. It is thought that these may reduce the blood supply to the liver parenchyma, resulting in atrophy of centrilobular hepatocytes in the areas furthest from the blood supply. Compensatory hyperplasia of periportal hepatocytes may then occur to produce the characteristic histological picture. ${ }^{89}$ In some cases larger nodules are formed which are visible macroscopically. In certain instances, vasculitic lesions have been reported in intrahepatic arteries. ${ }^{9}$ This could exacerbate zonal ischaemia and hence contribute to NRH.
DIAGNOSIS OF NRH

Histological abnormalities in NRH are often subtle and may be missed with conventional haemotoxylin and eosin stained sections leading to an underestimation of its true incidence. Reticulin staining, which demonstrates the sinusoidal framework of the liver is therefore most useful in demonstrating minor abnormalities of liver architecture and is advisable in all liver biopsies from patients with FS (fig 2). Because of the variable severity of changes within the liver, the abnormalities may be missed altogether by needle biopsy, a further reason for under-diagnosis. Wedge hepatic biopsy, with resultant sampling of a larger piece of tissue may be necessary. This allows an increased diagnostic yield but has obvious practical disadvantages.

PATHOPHYSIOLOGICAL CONSEQUENCES OF NRH The precise relationship between NRH and portal hypertension is as yet unclear. NRH is probably partly a secondary phenomenon occurring as a result of obliterative lesions in portal vein radicals which may partially obstruct portal blood flow. However, distortion or micro-architecture due to the alternating zones of hyperplastic lobules and compressed cell plates are likely to interfere with blood flow within the liver and may aggravate portal hypertension. ${ }^{9} 8$

INVESTIGATION OF PORTAL HYPERTENSION AND LIVER DISEASE IN FS

A full hepatic assessment in these patients should be performed before embarking on therapy, to tailor therapy individually to the patient. Even in patients with no gastrointestinal bleeding, assessment of portal hypertension and its complications is advisable before definitive therapy such as splenectomy is planned. Radiological procedures are of limited use in investigating liver disease in FS. There are no radiological features specific to NRH. However, radiological procedures may be helpful in excluding other pathological processes such as cirrhosis. If no varices are present in the oesophagus or stomach, then splenectomy can be performed without portosystemic shunting. If varices are confirmed, it is advisable to measure portal pressure, as in twin 2. WHVP measurement is the simplest and safest method of measuring pressure and is known to be an accurate measure of portal vein pressure in conditions such as alcoholic cirrhosis. In NRH the correlation is less certain and WHVP underestimates the directly measured portal vein pressure in some patients. If, in a given patient, serial portal pressure measurements are required to monitor therapy for portal hypertension, then initially both WHVP and portal pressure should be measured simultaneously and the information used to interpret subsequent readings obtained by WHVP measurement.

In our patient, the WHVP of $21 \mathrm{~mm} \mathrm{Hg}$ recorded three weeks before splenectomy and the directly measured portal vein pressure of 
$23 \mathrm{~mm} \mathrm{Hg}$ measured some six months later, after splenectomy, were remarkably similar. One would have expected a significant reduction in this pressure after subtracting splenic blood flow from the total portal blood flow. There are two possible explanations. Firstly, it may be that in our patient, unlike the few observations in previous reports, the portal vein pressure was not significantly diminished by splenectomy. The second possibility is that there was a significant degree of pre-sinusoidal block, so that the wedged hepatic pressure was an underestimate of portal vein pressure.

MANAGEMENT OF LIVER DISEASE IN FS

Various therapeutic strategies have been employed for the splenomegaly and neutropenia of Felty's syndrome, ranging from a wait and see approach, through treatment with second line or cytotoxic drugs, granulocyte-monocyte colony stimulating factor (GM-CSF), to splenectomy. ${ }^{10-12}$ If the management of patients with straightforward FS is still debatable, there is less debate about treatment once liver disease with portal hypertension has occurred. The presence of liver disease and portal hypertension would tend to bias towards procedures likely to reduce hepatic venous pressure.

There are a number of therapeutic options for treating portal hypertension in FS. It is known that oesophageal varices do not bleed if portal pressure does not exceed $12 \mathrm{~mm} \mathrm{Hg}$, and treatment should be especially considered when pressures are approaching this level. Propranolol has been widely used to prevent bleeding oesophageal varices in patients with cirrhosis, and it has been shown that a reduction of $>25 \%$ in WHVP as a response to propranolol is highly protective against further variceal bleeding. Splenectomy would be an obvious possibility. When splenectomy is planned, it is important to protect the patient against infection by bacteria with polysaccharide coats such as Streptococcus pneumoniae and Salmonella species. This may best be achieved by vaccination with Pneumovax (protecting against $S$ pneumoniae) before splenectomy, or daily penicillin $\mathrm{V}$ if pre-vaccination is not possible. However, if conservation of the spleen is required, then other porto-systemic anastomoses (for example, meso-caval shunts) may be selected to avoid injury to the massively enlarged spleen.

In the case of twin 2, the WHVP pressure was considerably greater than $12 \mathrm{~mm} \mathrm{Hg}$, with a consequent large risk of re-bleed and indicating that further therapy was indicated. Because she had NRH and therefore extremely well-preserved hepatocellular function, she was unlikely to be prone to hepatic encephalopathy after porto-systemic shunt procedures, unlike cirrhotic patients. Given this, and the excellent response to splenectomy in her identical twin, surgery was preferred to beta-blockade. Unfortunately, in contrast to her twin, twin 2 suffered from persistent bleeding from varices following her splenectomy. It would therefore have appeared advisable to have combined splenectomy with a spleno-renal shunt to decompress the portal system, and this lesson may be of use when considering operation in other patients with FS and varices.

\section{Conclusion}

Portal hypertension and NRH are uncommon but potentially fatal complications of FS. Optimal management requires a full hepatic work up, including venous pressure measurements. If hepatic biopsy is performed, reticulin staining is essential. Definitive therapy probably involves splenectomy combined with a shunting procedure to fully decompress the liver. Although the genetic background has a large role to play in the development of $\mathrm{NRH}$ in FS, environmental factors are also important, as illustrated by the two case histories.

POINTS

- Portal hypertension and NRH may be a serious complication of Felty's syndrome.

- Development of NRH results from a combination of genetic and environmental factors.

- In NRH and portal hypertension full hepatic investigation is required.

- Hepatic venous pressure measurements are indicated.

- Reticulin staining may be necessary to visualise NRH histologically.

- Definitive treatment may include both splenectomy and a shunting procedure.

1 Felty A R. Chronic arthritis in the adult associated with splenomegaly and leucopaenia. Fohns Hopkins Hosp Bull 1924; 35: 16

2 Campion G, Maddison P J, Goulding N, et al. The Felty Syndrome: a case-matched study of clinical manifestations and outcome, serologic features and immunogenetic associations. Medicine 1990; 69: 6979

3 Sansom D M, Bidwell J L, Maddison P J, Campion G, Klouda P T, Bradley B A. HLA DQ $\alpha$ and DQ $\beta$ restriction fragment length polymorphisms associated with Felty's syndrome and DR4-positive rheumatoid arthritis. Human Immunology 1987; 19: 269.

4 Lanchbury J S S, Jaeger E E M, Sansom D M, et al. Strong primary selection for the Dw4 subtype of DR4 accounts for the HLA-DQw7 association with Felty's syndrome. Human Immunology 1991; 32: 56.

5 Deighton C M, Walker D J, Griffiths I D, Roberts D F. The contribution of HLA to rheumatoid arthritis. Clinical genetics 1989; 36: 178 .

6 Breedveld F C, Lafeber G J M, Doekes G, Claas F H J, Cats A. Felty's syndrome: autoimmune neutropaenia or immune-complex mediated disease? Rheumatol International 1985; 5: 253-58.

7 Wanless I. Micronodular transformation (nodular regenerative hyperplasia) of the liver: a report of 64 cases among 2,500 autopsies and a new classification of benign hepatocellular nodules. Hepatology 1990; 11: 787-97.

8 Wanless I R, Godwin T A, Allen F, Feder A. Nodular regenerative hyperplasia of the liver in hematologic disorders: a possible response to obliterative portal venopathy. Medicine 1980; 59: 367-79.

9 Reynolds W J, Wanless I R. Nodular regenerative hyperplasia of the liver in a patient with rheumatoid vasculitis: a morphometric study suggesting a role for hepatic arteritis in the pathogenesis. F Rheumatol 1984; 11:

10 Dillon A M, Luthra H S, Conn D L, Ferguson R H. Parenteral gold therapy in the Felty syndrome. Medicine 1986; 65: 107-112.

11 Fiechtner J J, Miller D R, Starkenbaum G. Reversal of neutropaenia with methotrexate treatment in Felty's syndrome: Correlation of response with neutrophilreactive IgG. Arth Rheum 1989; 32: 194-200.

12 Markusse H M, Breedveld F C, Fibbe W E, Stern A C. Granulocyte-macrophage colony-stimulating factor treatment of neutropaenia associated with Felty's syndrome. Arth Rheum 1990; 33: 1865-7. 